\title{
Effects of multimodal communication program on patients with chronic aphasia: a single-subject A-B-A design study
}

\author{
Shohre Kaviani ${ }^{1}$, Afshin Samaei ${ }^{2}$, Masoomeh Salmani ${ }^{1}$, Noureddin Nakhostin Ansari ${ }^{3,4}$, Farnaz Dehnavi ${ }^{5}$, Ehsan \\ Shahverdi ${ }^{6}$
}

\footnotetext{
${ }^{1}$ Neuromuscular Rehabilitation Research Center, Semnan University of Medical Sciences, Semnan, Iran

${ }^{2}$ Neuromuscular Rehabilitation Research Center, Department of Internal Medicine, Semnan University of Medical

Sciences, Semnan, Iran

${ }^{3}$ Department of Physiotherapy, School of Rehabilitation, Tehran University of Medical Sciences, Tehran, Iran

${ }^{4}$ Sport Medicine Research Center, Neuroscience Institute, Tehran University of Medical Sciences, Tehran, Iran

${ }^{5}$ Semnan University of Medical Sciences, Semnan, Iran

${ }^{6}$ Blood Transfusion Research Center, High Institute for Research and Education in Transfusion Medicine, Tehran, Iran
}

\section{Type of article: Original}

\begin{abstract}
Background and aim: Aphasia as a common consequence of stroke, is an acquired neurologic communication disorder that can affect symbol language processing. Different types of intervention approaches have been introduced. Multimodal Communication Program (MCP) is a new augmentative alternative communication approach in chronic aphasia. The aim of this study was to investigate the effect of MCP on communication skills of patients with chronic aphasia.

Methods: This prospective, single subject, A-B-A design study was done during 2016 in Semnan, Iran. Participants were two patients with severe aphasia with a single left-hemisphere stroke. Three phases, including baseline, intervention and follow-up were administered. The patients received nine-hour intervention, over 10 working days.

Results: Three different scores were calculated for each patient: verbal efforts, the frequency of each modality and the accuracy of switching between modalities and the reaction time. The frequency of verbal modality increased for both patients. They could switch between modalities more successfully than before the intervention. The results for the reaction time, however were challenging. The onset reaction time decreased for patient 1 , and increased during switching between modalities, and patient 2 showed the opposite.

Conclusion: The MCP can improve the communication skills in patients with chronic post stroke aphasia. However, some factors, such as reduction of the patients' reaction time is probably related to the amount of allocated resources during intervention.

Trial registration: The trial was registered at IRCT center with ID: IRCT2016032325194N3.

Funding: The study was financed by Semnan University of Medical Sciences (Grant no.: A-10-333-3).

Keywords: Chronic aphasia, Stroke, Communication, Reaction time
\end{abstract}

\section{Introduction}

Aphasia, as an acquired neurogenic disorder, can affect symbolic processing of language skills (1). Such effects can have negative consequences on the patients' brain adequacy in planning, programming and the effective use of language skills (2). This is the story for about $21-38 \%$ of patients who have experienced acute stroke and are

\section{Corresponding author:}

Associate Professor Dr. Afshin Samaei, Neuromuscular Rehabilitation Research Center, Department of Internal Medicine, Semnan University of Medical Sciences, Semnan, Iran.

Tel: +98.2333654182, Email: afshinsamaei121@gmail.com

Received: September 23, 2017, Accepted: February 22, 2018, Published: March 2018

iThenticate screening: January 02, 2018, English editing: February 26, 2018, Quality control: March 10, 2018

This article has been reviewed / commented by four experts

(C) 2018 The Authors. This is an open access article under the terms of the Creative Commons Attribution-NonCommercialNoDerivs License, which permits use and distribution in any medium, provided the original work is properly cited, the use is non-commercial and no modifications or adaptations are made. 
struggling with aphasia (3-5). The epidemiology of stroke and its subtypes in the Middle East is unclear. Most previous studies have been performed in Western countries, and incidence rates are unlikely to apply in the Middle East (6). Treatment guidelines for aphasia recommend intensive speech and language therapy for chronic ( $\geq 6$ months) aphasia after stroke (7). These patients are mainly in middle or aging stages of their lives that need effective communication (8). However, patients' brains have tolerated serious injuries, and language disturbances will probably be the only permanent problem after stroke. However, the investigation of the existing studies on the language intervention for patients with severe aphasia is not promising; there are studies that showed language intervention for this group of patients did not reach satisfactory results (2). A recent research explained how speech and language therapy, especially during the acute phase of recovery can be help to re-establish language processing. Although, many studies showed that in patients with moderate to severe aphasia, language restoration was not possible $(9,10)$. Some of them suggested that instead of a uni-modal approach and insisting on language intervention for the patients with aphasia, therapists can apply a multimodal approach which includes a wide range of augmentative and alternative techniques $(11,12)$. There is evidence that Augmentative and Alternative Communication (AAC) can improve communication skills of patients with aphasia. However, therapists will face problems in teaching traditional AAC. In the traditional approach, the therapist teaches each modality separately, which is a time-consuming job. Patients have other problems such as disorders of executive function and memory, and switching between modalities can increase their problems. The possible consequence for the patient and the therapist will be some kind of disappointment and losing interest to move on (13-16). To solve these issues, Purdy and Van Dyke modified the traditional approach and presented a new insight to the multimodal training program (17). In Purdy's version of the multimodal training approach, there is a relationship between semantic concepts and different modalities to express them. But after stroke, this relationship disappears or can be weak, so patients are not able to use or switch between modalities automatically. The result is repetitive communication failures (17). Brain neuroplasticity studies indicated that recovery occurs through reorganization by the compensation neural network, even in patients with chronic aphasia. This reorganization in chronic phase will enable patients to learn the use of the new strategies to communicate when they are not able to have verbal communication (18). The AAC, as a compensatory approach, can improve long-term output in patients with aphasia. This approach gives patients opportunities to use residual language skills in communication and when a verbal tool has problems, the AAC can help to use other types of modalities to address communication problems (2). However, the efficacy of this new version of multimodal approach has not been investigated in patients with chronic aphasia in Persian language, the prevalence of chronic aphasia shows how many people may benefit if this approach gets approved. In the literature regarding Persian patients with aphasia however, few studies investigate the efficacy of language intervention, and there are none for the efficacy of the new multimodal approach. So, this study aimed to find out the effectiveness of MCP on Persian patients with chronic aphasia.

\section{Material and Methods}

\subsection{Research design}

This single-subject, A-B-A design project was carried out during 2016 in Semnan, Iran. Withdrawal designs or A-BA designs are characterized by at least one implementation and withdrawal of treatment over the course of the study. In an A-B-A design, a baseline phase (A) is followed by a treatment phase (B) and then by another baseline (A). These designs were developed to answer the important clinical question, "does this treatment work?" These designs provide good control of extraneous factors by enabling the researcher to determine whether the hypothesized change occurs each time the treatment is applied or withdrawn. The place for all evaluations and intervention was the university speech and language therapy clinic. The main variables were patients' efforts to use speech in the first confrontations, the frequency of different types of modalities, switching between the modalities and the patients' reaction time.

\subsection{Procedure}

This study had a single-subject, A-B-A design. The examiner administered the modality probe task during three phases including the baseline (three weeks, four sessions per week); then two weeks of multimodal communication program (three sessions per week); and finally, a follow-up phase (three weeks, one session per week). All these sessions were video recorded to double check the notes taken from the sessions. The examiner sent off information sheets and consent forms to the eligible participants who were attending the university clinics. Two consent forms were returned. The first author who is an experienced speech and language therapist evaluated the patients' language skills and defined the type of aphasia. Both patients had chronic aphasia, experienced one stroke on the left hemisphere, were right-handed, monolingual in Persian, without limb apraxia, and without obvious and interfering visual or hearing disorders. The patients did not have any history of drug abuse, mental illness, and recurrent stroke 
in the last six months. They had right hemiplegia so they had to use their left hand to write, to make gestures and to draw. They had intact auditory comprehension evaluated by the Mississippi test-Persian form. The Mississippi aphasia screening test (MAST) is a screening tool for quickly measuring the expressive and receptive language abilities in patients with aphasia. The expressive section of MAST included (Naming, automatic speech, repetition, yes/no accuracy, object recognition from a field of five, following verbal instructions, reading instructions, verbal fluency, and writing/spelling to dictation). The scores from each item of MAST are summed to produce sub-scale scores (receptive and expressive, each range 0-50). The scores from both sub-scales are summed to provide total score (range $0-100$ ). The MAST is a simple and brief measure, and time required to administer the MAST is $\sim 5-15$ min. The Persian version of the MAST is a valid and reliable instrument to assess patients with post-stroke aphasia (19). Their performance on the Mississippi test showed they had severe difficulty with naming tasks. Their speech output was featured by phonemic and verbal paraphasia. Their language output was one or two-word utterances, which was unintelligible because of paraphasia. They had difficulty to read and to write because of the brain damage. The participants had three scores. The first score was related to the first verbal attempts. In this section, the ten selected words, which were used in the probe in treatment, were provided to the patient during the assessment sessions and the patients were asked to express them in any way. If the patient, after presenting the image, selected a verbal modality for expression, one score would be considered, and if he did not choose a modality other than the modalities of speech, the patient would not receive a score. So, the patient can get a maximum score of ten and a minimum score of zero. The second score was the frequency and accuracy of the modality that means how many times patients transferred the concepts through any modality successfully (Switching between modalities). The scoring in the frequency of each modality was calculated as follows: The patient had ten opportunities for expressing the images, and were also randomly assigned 5 images, which were selected by the therapist. After expressing with the first modality, the patient replied to another modality. Therefore, there were 15 possibilities for expressing objective concepts. At the end of each session, the number of using of each modality was divided by the total number of opportunities, and the results were recorded. The switching score between the modalities was calculated so that after the presentation of each patient's first image and expression, the patient was asked to express that image with another modality. The ability to change between the modalities had one score and inability to change the modality. The maximum score for this part was ten and the minimum was zero. There was not an optimal level for the use of modalities, patients scored accurate even for their close efforts. For example, those verbal messages, including paraphasia scored correct as long as the original concept was there. The third score was related to reaction time. In the reaction time (RT), the interval between the presentation of the image to the patient and the patient's first attempt to express the image, regardless of the type of modality, was calculated with a stopwatch. After that, the therapist asked the patient to express the image by another modality, and again the time was calculated and recorded.

\subsection{Participant}

\subsubsection{Participant 1}

Patient 1 (P. 1) was a 64-year-old male who was retired from the army. He had non-fluent speech because of an ischemic cerebrovascular accident in the left middle cerebral artery eight years ago. His score on the Mississippi test was 26 out of 50 that showed a relatively preserved auditory comprehension. He did not show any sign of speech or oral apraxia. This patient was used to use pointing as the main tool to communicate.

\subsubsection{Participant 2}

Patient 2 (P. 2) was a 45-year-old male who had an ischemic stroke three years ago in the left frontal lobe. He had moderate apraxia of speech and oral according to the clinical observation. This patient used to use the conventional gestures to communicate. In both patients, the verbal output was non-fluent.

\subsection{Evaluation and Intervention Plans}

The general template of all the evaluation and intervention plan was taken from a previously published paper by Wallace et.al. The researchers of the present study were required to make some modifications to individualize the whole plan with the patients' needs.

\subsection{Probes}

The modality probe task included 10 high-frequency concepts in Persian (car, comb, telephone, camera, money, glass, book, watch, pencil, and key). These concepts were easy to gesture, draw, write and produce verbally. Each probe was a line drawing picture on a 12.5 by $22 \mathrm{~cm}$ card. Two sets of cards were available. One set was for the communication partner who was the research assistant, and was required to give feedback to the patients, or repair the conversation. The other set was handed in individually to the patients. The patients were also allowed to use a pen, a paper and a communication board. The examiner designed and made the communication board that included 
all 10 concepts in two rows, and each row included five pictures. Its dimensions were 28 by $21.50 \mathrm{~cm}$. Each time, the patients were required to try to transfer one concept through any possible way (pointing to the communication board, naming, making a gesture, drawing, and writing) to the partner within one minute. When the patients were able to convey concepts to their communication partners with any modality, the examiner scored the accuracy and the type of the modality in a table. The evaluation of patients' semantic knowledge regarding the naming test (Nilipour 2011) took place in the second session of the baseline assessment. The naming test includes 50 pictures of different objects. These pictures were presented in ten rows with five items each. The Referential Communication Task (RCT) happened in the third session. This task includes the mentioned 10 high frequency concepts in context. These pictures depict a person who uses the target concept (ex. a man uses the camera). In the picture, the target concepts were bold. The participants should ask for the matched card through any possible modality. To check patients' abilities in switching between modalities, the communication partners pretended they did not understand the concepts (at least five times) and presented a wrong card. So, the patients should choose another modality. Patients were scored on successful switching regardless of the types of modalities (verbally or non-verbally). The last factor that the examiner evaluated was the patients' reaction time. In this study, the reaction time is defined as the time between presenting a sensory stimulation and participant's response. In clinical settings, reaction time is important and beneficial because it can show the language and mental processing. Two types of reaction time were recorded, one for confrontational naming and the other one was related to switching modalities.

\subsection{Treatment phase}

An intensive intervention plan that includes nine sessions of MCP over two weeks (five working days) was applied. In each one-hour session, the therapist presented all 10 concepts through all five modalities. This part of the intervention was according to the errorless approach to stop patients from unsuccessful efforts. The patients were required to imitate all these concepts after the therapist. The therapist helped patients during the imitation. Cues or help was decreased when the patients' skills improved with the use of different modalities. At the end of the imitation part, the therapist reviewed the concepts through all five modalities and reminded the patients that they were allowed to use all five modalities to convey each concept. At the beginning of the odd sessions $\left(3^{\text {rd }}, 5^{\text {th }}, 7^{\text {th }}\right.$, and $\left.9^{\text {th }}\right)$, the therapist did a pretest on patients' skills to switch between modalities and to name pictures through any possible way.

\subsection{Follow-up}

The week after the treatment phase, the follow-up phase was started for three weeks, and each week was considered as one session. In these sessions, the patients answered the probe modality task. The second session had an extra task, which was the RCT.

\subsection{Ethics of research:}

Individuals were asked to sign an informed consent form before blood samples were obtained. All the terms of the Helsinki declaration were considered, and personal information remained anonymous. The study was approved by the ethics committee of Semnan University of Medical Sciences, Semnan, Iran and was also registered at the Iranian Registry of Clinical Trials (IRCT) with the unique ID of IRCT2016032325194N3.

\section{Results}

\subsection{First Verbal Attempts}

The participants had chronic aphasia and it would be desirable to expect they were able to use speech for naming at the first confrontation. Table 1 shows that in the baseline phase, the use of speech for P.1 was zero, but P.2 used speech twice in 10 opportunities. These figures increased during the treatment phase and reached to almost three times in 10 opportunities in the follow-up phase for both patients.

\subsection{The Frequency of Different Modalities}

The frequencies of each modality in 10 concepts for each patient were presented in Figure 1 and Figure 2. Patients 1 used mainly the communication board and speech while patients 2 relied on gestures, communication board and the speech in all three phases. Regarding to the phase of the intervention plan, in the follow-up, the patients showed better use of different types of modalities than the other two phases. 


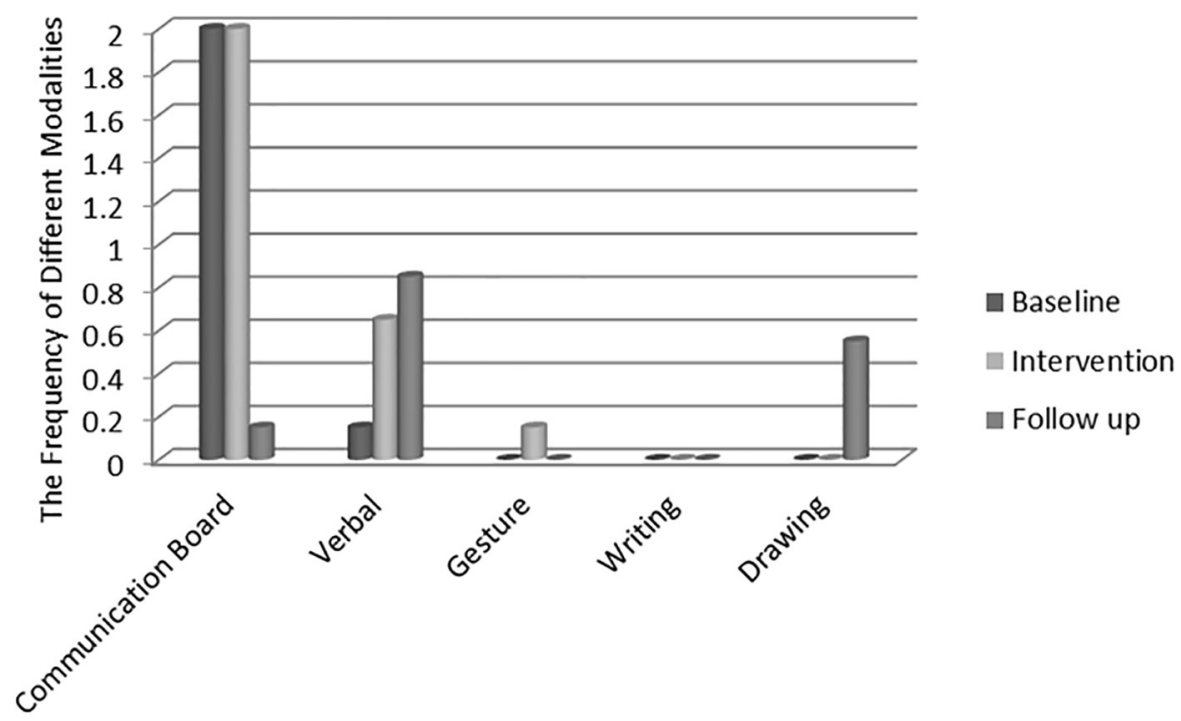

Figure 1. The Frequency of each Modality in Different Phases for Patient 1

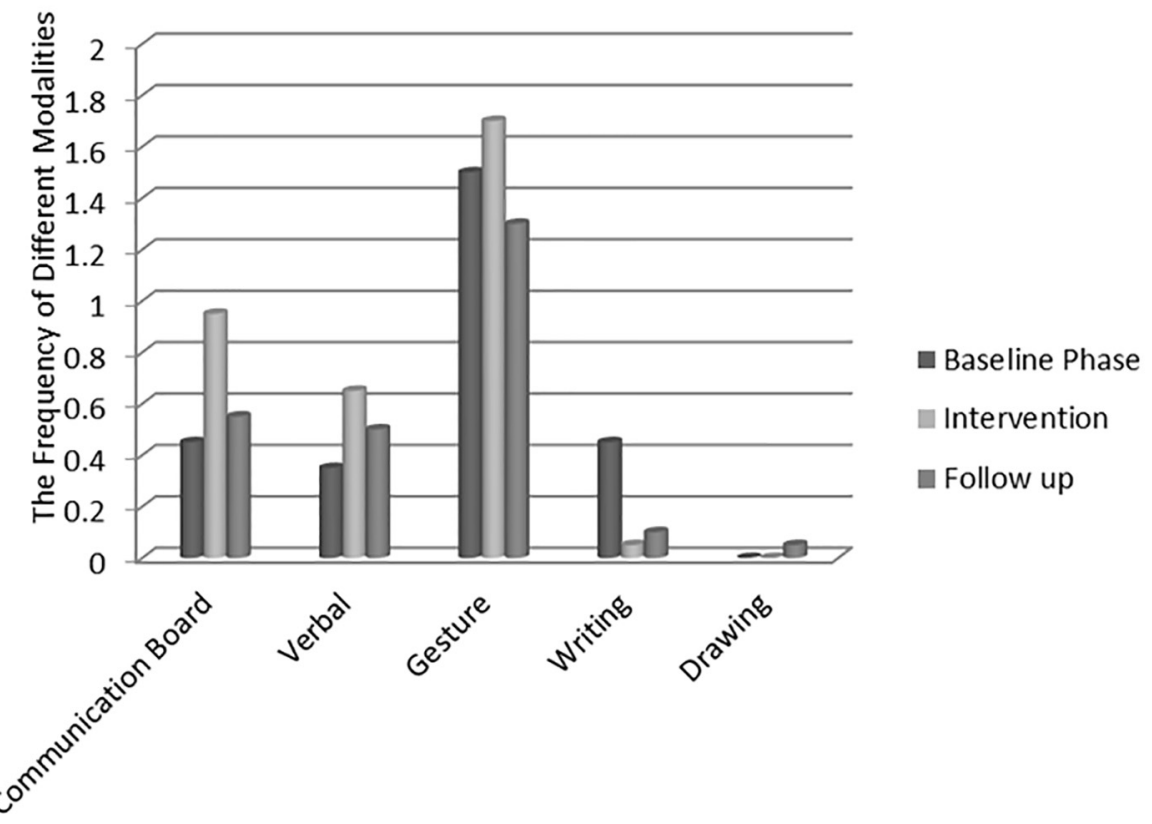

Figure 2. The Frequency of each Modality in Different Phases for Patient 2

\subsection{The Switching between Modalities}

The patients were able to switch accurately between modalities from the first sessions even though their success rates were below $50 \%$. The accuracy rates increased to over $90 \%$ in the treatment phase and follow-up for P1 and reached to $70 \%$ for P2 (Table 2).

\subsection{The Reaction Time}

In the present study, two types of stimulation were considered. The first one was related to the time between presenting the picture and the patients' response through any possible way. The second reaction time was related to the time between SLP request to change the modality and the patients' response. The P.1 showed an obvious decline in his first reaction time, but P.2 showed a marginal increase. This situation was reversed in reaction time to switch between modalities with a major peak in the follow-up stage for P.1 (Table 3). 
Table 1. The first verbal attempts score

\begin{tabular}{|l|l|l|l|l|l|l|}
\hline \multirow{2}{*}{ Session } & \multicolumn{2}{|l|}{ Baseline } & \multicolumn{2}{l|}{ Intervention } & \multicolumn{2}{l|}{ Follow-up } \\
\cline { 2 - 7 } & P. 1 & P. 2 & P. 1 & P. 2 & P. 1 & P. 2 \\
\hline 1 & 0 & 2 & & & & \\
\hline 2 & 0 & 1 & & & & \\
\hline 3 & 0 & 2 & & & & \\
\hline 4 & 0 & 2 & & & & \\
\hline 5 & & & 0 & 2 & & \\
\hline 6 & & & 1 & 2 & & \\
\hline 7 & & & 2 & 2 & & \\
\hline 8 & & & 1 & 4 & & \\
\hline 9 & & & & & 3 & 4 \\
\hline 10 & & & & & 1 & 2 \\
\hline 11 & & & & & 3 & 3 \\
\hline $\mathrm{M}$ & 0 & 1.75 & 1.75 & 1.75 & 2.33 & 3 \\
\hline
\end{tabular}

Table 2. The Accuracy to Change the Modalities

\begin{tabular}{|l|l|l|l|l|l|l|}
\hline \multirow{2}{*}{ Session } & \multicolumn{2}{|l|}{ Baseline } & \multicolumn{2}{l|}{ Intervention } & \multicolumn{2}{l|}{ Follow-up } \\
\cline { 2 - 7 } & P. 1 & P. 2 & P. 1 & P.2 & P. 1 & P. 2 \\
\hline 1 & 0 & 2 & & & & \\
\hline 2 & 1 & 5 & & & & \\
\hline 3 & 2 & 5 & & & & \\
\hline 4 & 1 & 6 & & & & \\
\hline 5 & & & 2 & 7 & & \\
\hline 6 & & & 3 & 8 & & \\
\hline 7 & & & 5 & 6 & & \\
\hline 8 & & & 6 & 7 & & \\
\hline 9 & & & & & 9 & 8 \\
\hline 10 & & & & & 9 & 7 \\
\hline 11 & & & & & 10 & 6 \\
\hline $\mathrm{M}$ & 1 & 4.5 & 4 & 7 & 9.333 & 7 \\
\hline
\end{tabular}

Table 3. The reaction time for pictures and the reaction time to switch between modalities

\begin{tabular}{|c|c|c|c|c|c|c|}
\hline \multirow[t]{2}{*}{ Session } & \multicolumn{2}{|c|}{$\begin{array}{l}\text { Baseline } \\
\text { (Pictures/modalities) }\end{array}$} & \multicolumn{2}{|c|}{$\begin{array}{l}\text { Intervention } \\
\text { (Pictures/modalities) }\end{array}$} & \multicolumn{2}{|c|}{$\begin{array}{l}\text { Follow-up } \\
\text { (Pictures/modalities) }\end{array}$} \\
\hline & P. 1 & P. 2 & P. 1 & P. 2 & P. 1 & P. 2 \\
\hline 1 & $49 / 71$ & $33 / 73$ & & & & \\
\hline 2 & $33 / 71$ & $26 / 21$ & & & & \\
\hline 3 & $37 / 125$ & $27 / 26$ & & & & \\
\hline 4 & $38 / 74$ & $28 / 16$ & & & & \\
\hline 5 & & & $33 / 53$ & $27 / 10$ & & \\
\hline 6 & & & $34 / 53$ & $31 / 27$ & & \\
\hline 7 & & & $26 / 74$ & $28 / 25$ & & \\
\hline 8 & & & $30 / 119$ & $32 / 17$ & & \\
\hline 9 & & & & & $27 / 345$ & $36 / 26$ \\
\hline 10 & & & & & $28 / 412$ & $26 / 17$ \\
\hline 11 & & & & & $27 / 384$ & $29 / 10$ \\
\hline $\begin{array}{l}\text { Mean } \\
\text { (Pictures/modalities) }\end{array}$ & $39.25 / 85.25$ & $28.5 / 34$ & $30.75 / 74.75$ & $29.5 / 19.75$ & $27.33 / 380.33$ & $30.33 / 17.66$ \\
\hline
\end{tabular}

\section{Discussion}

This study aimed to investigate the effectiveness of MCP on communication skills in patients with chronic aphasia. The present study is the first formal report on the effect of the MCP on naming abilities of patients with aphasia post 
stroke. The discussion focuses on how features of this particular protocol might have influenced the first verbal attempts, the frequency and switching between modalities and the reaction time. We designed this study as a single-subject, A-B-A design study. The major criticism of aphasia trials is that they do not take into account aphasic and treatment heterogeneity. The single case approach, well executed, is specific about the underlying deficit being treated, and equally specific about the treatment given. Such specificity will make it possible to predict which other patients should benefit from such treatment, although at the moment, replications are few and far between. Even Wertz (1992) acknowledges that it is the single case treatment study which will provide information about specific treatment effects. Judging whether a good recovery has taken place in aphasia is rather more difficult than it is in the case of most medical problems. Since, in practice, patients are treated on the basis of their particular combination of language deficits, the single subject approach has many clinical advantages. Chief amongst these is the fact that it can be demonstrated to the patients themselves that the therapy is working. The approach also makes it possible to look at the size of the improvement in that particular case and its functional importance (20). Single subject designs are often confused with clinical case reports or case studies. The two are very different. The case report or case study is a description of a course of treatment of an individual. The case report is therefore a nonexperimental form of inquiry. Single subject, in contrast, include controlled manipulation of an independent variable and are conducted prospectively. The results of this study showed that Multimodal communication programs over a short time have positive effects on patients' verbal skills. The findings support the idea that MCP as an AAC strategy, increases the use of residual verbal output (21). In consistent with the brain neuroplasticity studies; this study demonstrated that even in chronic phase of aphasia, learning occurred (18). Some studies have reported that people with aphasia benefit from informational redundancy, and comprehend better when information is presented through multiple modalities rather than a single modality, additionally, with all type modalities for expressing concepts to be learned, patients can use them to compensate communication challenges $(2,22)$. The MCP has these properties, and one of our purposes in this study was to investigate the frequency of each of the learned modalities.

Whereas most people without communication challenges do not routinely use drawing for their communication attempts, many people with aphasia do not automatically recognize the potential for communicative drawing and do not spontaneously use drawing to convey ideas $(23,24)$. The MCP provided direct instruction to encourage its use. The frequency of drawing in this study, for both patients, increased after the intervention. The increase might be because the therapist provided an extra amount of time during communication and helped patients reduce their reliance on linguistic symbols. So, they were able to convey their intended meaning through drawing. The score of pointing to the communication board for the first patient was more than patient 2 in baseline phase. High scores may be because of facilitated communicative interactions with people who are not familiar with aphasia, and persons with aphasia do not require symbolic or linguistic processing beyond the person's capabilities (2). These scores for the first patient decreased, but for the second patient, increased during follow up phase. Reduction in scores of pointing for P.1 may be a result of improvement in the use of other modalities such as speech. Increased score for patient 2 after intervention, may be due to the feature of easy access of pointing for conveying messages. The score of writing modality for P.1 was zero in all three phases and P.2 showed a reduction in the use of writing. This might be a consequence of their paralysis and the nature of brain function during writing. Gestures are a standard supplement to most people's natural speech production. Although some people with aphasia retain some spontaneous use of gestures as supplements to speak, most struggle with using gestures, comparable to their challenges with other forms of linguistic and symbolic systems (2). Higher score for the use of gestures for P.2 than that of P.1 was probably for easy access to actionable information requisite for gesture. At the end of intervention both patients used gestures less than before, which might be a consequence of the increase in verbal output. This program, with its special features, can improve patients' abilities to switch between modalities and then reduce the number of communication failures. Hence, increased score of switching between modalities, in this study, demonstrated that patients were able to use different modalities for communication goals. This finding is consistent with the results presented by prior studies $(22,25)$. The use of alternative communication modalities requires access to different types of semantic information that we include both patients have nearly normal semantic processing. The results of the present study demonstrated that both participants showed an increase in the frequency and accuracy of each modality after intervention by the MCP. These findings are similar to Purdy and Wallace's studies (17, 22). These researchers showed the integrated MCP can improve the use of different modalities in acute and chronic phases. However, this finding is inconsistent with Purdy and Youshihsta's findings (13, 14). These differences were due to the design of their studies. These prior studies, all modalities of one concept do not instruct simultaneously and integratory, thus do not create linkage between linguistic concepts and all modalities for expressing them. Thus, when the modalities remain separate from the linguistic system, their usage of alternative modes of expression may not generalize. In order to switch, when an initial communicative attempt is unsuccessful, individuals must 
consciously inhibit the natural tendency to repeat the first modality and purposely initiate an alternative response. One of the novel findings in the current study is the reduction of the reaction time that happened differently for each patient. The latency for the first effort decreased for P.1 and increased for P.2. Inversely, the latency for switching between modalities increased for P.1 and decreased for P.2. The use of different types of modalities and the number of allocated resources for each patient might be the key explanation. The P.1 used pointing while P.2 used other types of modalities that were more time consuming. In the switching between modalities P.2 was more successful than P.1 because he had more than one modality available to use compared to P.1.

\section{Conclusions}

In summary, MCP, with its characteristics, can improve communication skills of chronic aphasic patients. However, we suggest further investigation to determine efficacy of MCP.

\section{Trial registration:}

The trial was registered at IRCT center with ID: IRCT2016032325194N3.

\section{Acknowledgments and funding:}

The authors thank Semnan University of Medical Sciences for their financial support (Grant A-10-333-3).

\section{Conflict of Interest:}

There is no conflict of interest to be declared.

\section{Authors' contributions:}

All authors contributed to this project and article equally. All authors read and approved the final manuscript.

\section{References:}

1) Hallowell B. Aphasia and other acquired neurogenic language disorders: A guide for clinical excellence: Plural Publishing; 2016.

2) Chapey R, Hallowell B. Introduction to language intervention strategies in adult aphasia. Language intervention strategies in aphasia and related neurogenic communication disorders. 2001; 4: 3-8.

3) Brust J, Shafer SQ, Richter RW, Bruun B. Aphasia in acute stroke. Stroke. 1976; 7(2): 167-74. doi: 10.1161/01.STR.7.2.167. PMID: 1265809.

4) Wade DT, Hewer RL, David RM, Enderby PM. Aphasia after stroke: natural history and associated deficits. J Neurol Neurosurg Psychiatry. 1986; 49(1): 11-6. doi: 10.1136/jnnp.49.1.11. PMID: 2420939, PMCID: PMC1028640.

5) Pedersen PM, Jørgensen HS, Nakayama H, Raaschou HO, Olsen TS. Aphasia in acute stroke: incidence, determinants, and recovery. Ann Neurol. 1995; 38(4): 659-66. doi: 10.1002/ana.410380416. PMID: 7574464.

6) Azarpazhooh MR, Etemadi MM, Donnan GA, Mokhber N, Majdi MR, Ghayour-Mobarhan M, et al. Excessive incidence of stroke in Iran: evidence from the Mashhad Stroke Incidence Study (MSIS), a population-based study of stroke in the Middle East. Stroke. 2010; 41(1): e3-e10. doi: 10.1161/STROKEAHA.109.559708. PMID: 19926844.

7) Breitenstein C, Grewe T, Flöel A, Ziegler W, Springer L, Martus P, et al. Intensive speech and language therapy in patients with chronic aphasia after stroke: a randomised, open-label, blinded-endpoint, controlled trial in a health-care setting. The Lancet. 2017; 389(10078): 1528-38. doi: 10.1016/S0140-6736(17)300673.

8) Moreaud O, David D, Brutti-Mairesse MP, Debray M, Mémin A. [Aphasia in elderly patients Psychol Neuropsychiatr Vieil. 2010; 8(1): 43-51. doi: 10.1684/pnv.2009.0185. PMID: 20215098.

9) LaPointe LL. Foundations: Adaptation, accommodation, Aristos. 2005.

10) LaPointe LL. Aphasia and related neurogenic language disorders: Thieme; 2005.

11) Hallowell B, Chapey R, Chapey R. Language intervention strategies in aphasia and related neurogenic comunication disorders. Philadelphia: Lippincott, Williams, \& Wilkins; 2008.

12) Rose ML, Raymer AM, Lanyon LE, Attard MC. A systematic review of gesture treatments for post-stroke aphasia. Aphasiology. 2013; 27(9): 1090-127. doi: 10.1080/02687038.2013.805726.

13) Purdy MH, Duffy RJ, Coelho CA. An investigation of the communicative use of trained symbols following multimodality training. Clinical aphasiology. 1994; 22: 345-56. 
14) Yoshihata $H$, Watamori $T$, Chujo $T$, Masuyama $K$. Acquisition and generalization of mode interchange skills in people with severe aphasia. Aphasiology. 1998; 12(12): 1035-45. doi: 10.1080/02687039808249468.

15) Purdy M. Executive function ability in persons with aphasia. Aphasiology. 2002; 16(4): 549-57. doi: 10.1080/02687030244000176.

16) Purdy M, Koch A. Prediction of strategy usage by adults with aphasia. Aphasiology. 2006; 20(2-4): 33748. doi: 10.1080/02687030500475085.

17) Purdy M, Van Dyke JA. Multimodal communication training in aphasia: A pilot study. J Med Speech Lang Pathol. 2011; 19(3): 45. PMID: 24558295, PMCID: PMC3927416.

18) Godecke E. Efficacy of aphasia therapy in the acute setting: Curtin University of Technology. 2008.

19) Khatoonabadi AR, Nakhostin-Ansari N, Piran A, Tahmasian H. Development, cross-cultural adaptation, and validation of the Persian Mississippi Aphasia Screening Test in patients with post-stroke aphasia. Iran J Neurol. 2015; 14(2): 101-7. PMID: 26056555, PMCID: PMC4449390.

20) Franklin S. Designing single case treatment studies for aphasic patients. Neuropsychological Rehabilitation. 1997; 7(4): 401-18. doi: 10.1080/713755544.

21) Fried-Oken M, Beukelman DR, Hux K. Current and future AAC research considerations for adults with acquired cognitive and communication impairments. Assistive Technol. 2012; 24(1): 56-66. doi: 10.1080/10400435.2011.648713. PMID: 22590800, PMCID: PMC3760684.

22) Wallace SE, Purdy M, Skidmore E. A multimodal communication program for aphasia during inpatient rehabilitation: A case study. NeuroRehabilitation. 2014; 35(3): 615-25. doi: 10.3233/NRE-141136. PMID: 25227547, PMCID: PMC4216742.

23) Lyon JG. Communicative drawing: An augmentative mode of interaction. Aphasiology. 1995; 9(1): 84-94. doi: 10.1080/02687039508248694.

24) Lyon JG. Drawing: Its value as a communication aid for adults with aphasia. Aphasiology. 1995; 9(1): 33 50. doi: 10.1080/02687039508248687.

25) Simmons-Mackie NN, Damico JS. Reformulating the definition of compensatory strategies in aphasia. Aphasiology. 1997; 11(8): 761-81. doi: 10.1080/02687039708250455. 